1. Farquhar MG. The glomerular basement membrane: not gone, just forgotten. J Clin Invest. 2006; 116(8):2090-2093.

2. Garg P, Holzman LB. Podocytes: gaining a foothold. Exp Cell Res. 2012;318(9):955-963.

3. Reeves W, Caulfield JP, Farquhar MG. Differentiation of epithelial foot processes and filtration slits: sequential appearance of occluding junctions, epithelial polyanion, and slit membranes in developing glomeruli. Lab Invest. 1978;39(2):90-100.

4. Farquhar MG, Vernier RL, Good RA. An electron microscope study of the glomerulus in nephrosis, glomerulonephritis, and lupus erythematosus. J Exp Med. 1957;106(5):649-660.

5. Shirato I, Sakai T, Kimura K, Tomino Y, Kriz W. Cytoskeletal changes in podocytes associated with foot process effacement in Masugi nephritis. AmJ Pathol. 1996;148(4):1283-1296.

6. Kaplan JM, et al. Mutations in ACTN4, encoding alpha-actinin-4, cause familial focal segmental glomerulosclerosis. Nat Genet. 2000;24(3):251-256.

7. Faul C, Asanuma K, Yanagida-Asanuma E, Kim K, Mundel P. Actin up: regulation of podocyte structure and function by components of the actin cytoskeleton. Trends Cell Biol. 2007;17(9):428-437.

8. Verma R, Kovari I, Soofi A, Nihalani D, Patrie K, Holzman LB. Nephrin ectodomain engagement results in Src kinase activation, nephrin phosphorylation, Nck recruitment, and actin polymerization. J Clin Invest. 2006;116(5):1346-1359.
9. Soda $\mathrm{K}$, et al. Role of dynamin, synaptojanin, and endophilin in podocyte foot processes. J Clin Invest. 2012;122(12):4401-4411.

10. Reiser J, Kriz W, Kretzler M, Mundel P. The glomerular slit diaphragm is a modified adherens junction. J Am Soc Nephrol. 2000;11(1):1-8.

11. Fukasawa H, Bornheimer S, Kudlicka K, Farquhar MG. Slit diaphragms contain tight junction proteins. J Am Soc Nephrol. 2009;20(7):1491-1503.

12. Caulfield JP, Reid JJ, Farquhar MG. Alterations of the glomerular epithelium in acute aminonucleoside nephrosis. Evidence for formation of occluding junctions and epithelial cell detachment. $L a b$ Invest. 1976;34(1):43-59.

13. McMahon HT, Boucrot E. Molecular mechanism and physiological functions of clathrin-mediated endocytosis. Nat Rev Mol Cell Biol. 2011;12(8):517-533.

14 . Sever $\mathrm{S}$, et al. Proteolytic processing of dynamin by cytoplasmic cathepsin $\mathrm{L}$ is a mechanism for proteinuric kidney disease. J Clin Invest. 2007; 117(8):2095-2104.

15. Taylor MJ, Lampe M, Merrifield CJ. A feedback loop between dynamin and actin recruitment during clathrin-mediated endocytosis. PLoS Biol. 2012;10(4):e1001302.

16. de Beco S, Gueudry C, Amblard F, Coscoy S. Endocytosis is required for $\mathrm{E}$-cadherin redistribution at mature adherens junctions. Proc Natl Acad Sci US A. 2009;106(17):7010-7015.

17. Matsuda M, Kubo A, Furuse M, Tsukita S. A pecu- liar internalization of claudins, tight junctionspecific adhesion molecules, during the intercellular movement of epithelial cells. J Cell Sci. 2004; 117(pt 7):1247-1257.

18. Takahashi S, et al. The E3 ubiquitin ligase LNX1p 80 promotes the removal of claudins from tight junctions in MDCK cells. J Cell Sci. 2009;122(pt 7):985-994.

19. Ivanov AI, Nusrat A, Parkos CA. Endocytosis of the apical junctional complex: mechanisms and possible roles in regulation of epithelial barriers. Bioessays. $2005 ; 27(4): 356-365$.

20. Levayer R, Pelissier-Monier A, Lecuit T. Spatial regulation of Dia and Myosin-II by RhoGEF2 controls initiation of E-cadherin endocytosis during epithelial morphogenesis. Nat Cell Biol. 2011;13(5):529-540.

21. Brasch J, Harrison OJ, Honig B, Shapiro L. Thinking outside the cell: how cadherins drive adhesion. Trends Cell Biol. 2012;22(6):299-310.

22. Gavard J, Gutkind JS. VEGF controls endothelialcell permeability by promoting the beta-arrestindependent endocytosis of VE-cadherin. Nat Cell Biol. 2006;8(11):1223-1234.

23. de Beco S, Gueudry C, Amblard F, Coscoy S. Endocytosis is required for E-cadherin redistribution at mature adherens junctions. Proc Natl Acad Sci US A. 2009;106(17):7010-7015.

24. Klink TA, et al. Development and validation of a genericfluorescent methyltransferase activity assay based on the transcreener AMP/GMP assay. J Biomol Screen. 2012;17(1):59-70.

\title{
Leaky channels make weak muscles
}

\section{Alfred L. George Jr.}

Departments of Medicine and Pharmacology, Vanderbilt University, Nashville, Tennessee, USA.

\begin{abstract}
Mutations in the skeletal muscle voltage-gated calcium channel (Cav1.1) have been associated with hypokalemic periodic paralysis, but how the pathogenesis of this disorder relates to the functional consequences of mutations was unclear. In this issue of the JCI, Wu and colleagues recapitulate the disease by generating a novel knock-in Cav1.1 mutant mouse and use this model to investigate the cellular and molecular features of pathogenesis. They demonstrated an aberrant muscle cell current conducted through the $\mathrm{Ca}_{v} 1.1$ voltage-sensor domain (gating pore current) that explains an abnormally depolarized muscle membrane and the failure of muscle action potential firing during challenge with agents known to provoke periodic paralysis. Their work advances understanding of molecular and cellular mechanisms underlying an inherited channelopathy.
\end{abstract}

Ion channels are ubiquitous membrane proteins that confer selective ionic permeability to the plasmalemma or intracellular membranes and enable a wide variety of important physiological processes, including membrane excitability, synaptic transmission, signal transduction, cell volume regulation, and transcellular ion transport. The vital nature of ion channels is reflected by the existence of inherited disorders

Conflict of interest: The author has declared that no conflict of interest exists.

Citation for this article: J Clin Invest. 2012; 122(12):4333-4336. doi:10.1172/JCI66535. caused by mutations in genes that encode these proteins (1-5). These "channelopathies" represent more than 50 human genetic diseases, including several affecting skeletal muscle contraction, such as the periodic paralyses and nondystrophic myotonias (see "Muscle channelopatbies").

\section{Hypokalemic periodic paralysis}

Plasma membrane channels in skeletal muscle are essential for the generation and propagation of action potentials, leading to release of intracellular calcium through the process of excitation-contraction coupling. Ion channel dysfunction can hinder contraction by impairing action potential firing along the membrane. A characteristic symptom of this phenomenon is known as "periodic paralysis," a form of paroxysmal weakness that occurs in the absence of neuromuscular junction or motor neuron disease. Periodic paralysis is most often thought of as an inherited disease, but certain acquired conditions can produce a similar phenotype. Disturbances in plasma potassium ion concentration often accompany bouts of weakness, and the direction of change has been used to classify the condition as hypokalemic, hyperkalemic, or normokalemic periodic paralysis (6). These conditions are not lethal, because respiratory muscles are spared.

Although the clinical features and inheritance pattern have been known since the first half of the twentieth century, the pathophysiology of periodic paralysis remained mysterious until approximately 30 years ago, when investigators in Germany published their electrophysiological observations on explanted intercostal muscle fibers from subjects with periodic paralysis (7). These studies revealed that resting membrane potential was less nega- 


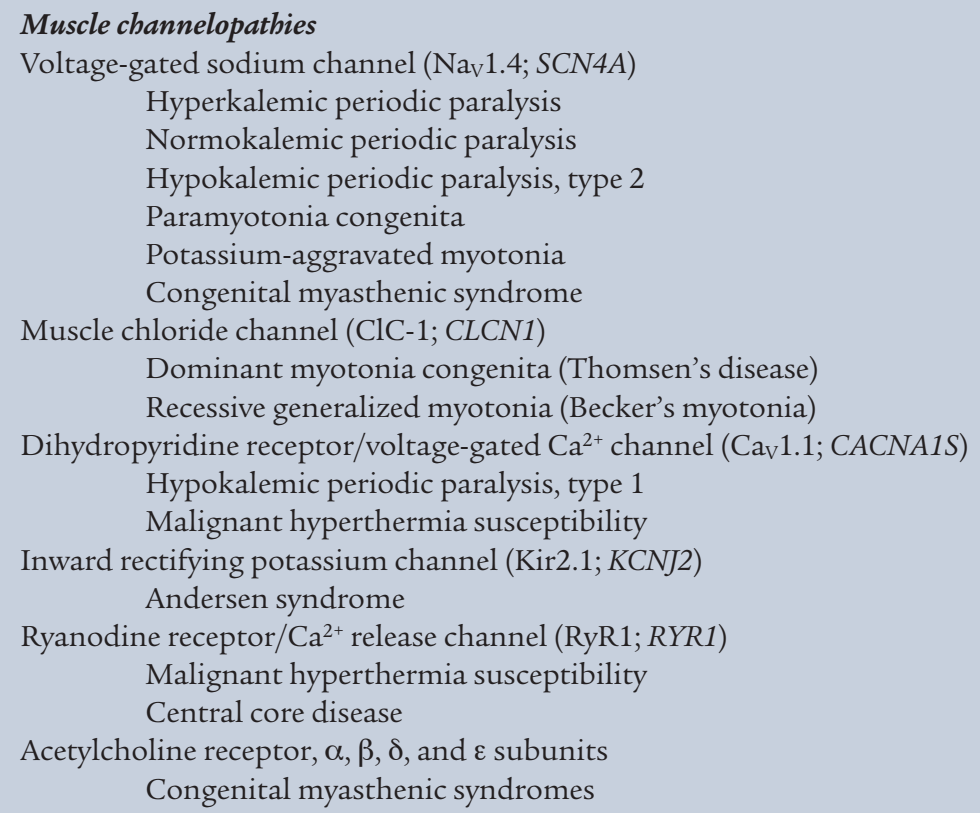

tive in fibers from hypokalemic periodic paralysis (HypoPP) subjects compared with normal muscle. Further, exposure of the fibers to low extracellular potassium concentration evoked a further depolarization, whereas this condition hyperpolarized normal muscle membranes. A similar paradoxical depolarization was observed after application of insulin. Both hypokalemia and insulin treatment rendered the fibers electrically inexcitable, owing to inactivation of voltage-gated sodium channels by the strongly depolarized membrane potential. By contrast, muscle fibers from subjects with hyperkalemic periodic paralysis (HyperPP) had normal resting membrane potentials but exhibited depolarization and inexcitability when exposed to elevated extracellular potassium (8). Tetrodotoxin, a highly specific blocker of voltage-gated sodium channels, reversed the depolarization occurring in HyperPP fibers but had no effect on HypoPP muscle. These observations established fundamental features of the pathogenesis of periodic paralysis but left unanswered key questions about the nature of the depolarizing stimulus in the hypokalemic variant.

Advances in the molecular genetics of periodic paralysis revealed that HyperPP is caused exclusively by mutations in SCN4A, the gene encoding the skeletal muscle voltage-gated sodium channel ( $\left.\mathrm{Na}_{V} 1.4\right)$, whereas HypoPP is caused most often by mutations in CACNA1S, encoding the pore- forming subunit of an L-type voltage-gated calcium channel $\left(\mathrm{Ca}_{V} 1.1\right)$, and less commonly by SCN4A mutations (2). Although both syndromes are associated with notable allelic heterogeneity, CACNA1S and SCN4A mutations associated with HypoPP are clustered within voltage-sensor domains. A brief digression on the topic of voltage-gated channel structure and function will help clarify the pathophysiological significance of this mutation clustering.

\section{Voltage-gated channel structure and function}

The sodium and calcium channels expressed in nerve, heart, and muscle belong to a superfamily of ion channels that are gated (opened and closed) by changes in membrane potential (9). The main pore-forming $\alpha$-subunits have a four-fold symmetry consisting of structurally homologous domains (D1-D4), each containing four transmembrane segments that comprise the voltagesensor domain (S1-S4) and a separate pore domain (S5-S6), important for determining ion selectivity (Figure 1A). The S4 segment, which functions as the main voltage-sensing element, is amphipathic, with basic amino acids (arginine or lysine) at every third position surrounded by hydrophobic residues. Activation of voltage-gated channels is evoked by a membrane depolarization that acts to propel the S4 segments in an outward direction away from the negative electrostatic cell interior. Subsequent con- formational changes involving the S6 segment open the ion pore and permit rapid movement of ions through a passageway created by the pore domain.

The highly conserved S4 segment has received an enormous amount of attention for the past two decades, particularly with regard to the molecular motions that carry its positive charges through the membrane electric field $(10,11)$. One startling revelation regarding sodium and potassium channels was that the S4 segment becomes accessible to aqueous protein-modifying reagents during gating motions $(12,13)$. This observation led to the hypothesis that S4 segments travel through the membrane via a water-filled cavity. Even more intriguing was the observation that histidine substitutions for arginine residues within the S4 segment generate a proton pore that is separate from the main ion permeation pathway in the pore domain (14). The current flowing through the voltage-sensor pore (also known as the gating pore) was termed the "omega" or "gating pore" current (Figure 1B).

Because these S4 segment histidine substitutions created unnatural channels, astute researchers investigating the functional consequences of channelopathyassociated mutations recognized that this mechanism might explain the pathophysiology of HypoPP. Specifically, sodium channel mutations associated with HypoPP that replace S4 segment arginine residues create channels that conduct an anomalous inward current at resting membrane potentials $(15-18)$. The in vivo relevance of this mechanism was demonstrated subsequently using a mouse model of the disease ( $\mathrm{Na}_{V} 1.4 \mathrm{R} 669 \mathrm{H}$ knock-in), in which an anomalous inward current was detected in muscle cells at hyperpolarized potentials (19). These investigations offered a molecular explanation for HypoPP caused by sodium channel mutations, but did not address what happens with the more common calcium channel mutations. Furthermore, prior studies of mutations engineered in human $\mathrm{Ca}_{\mathrm{V}} 1.1$ did not reveal a consistent and compelling pattern of channel dysfunction that would explain the phenotype, in part because of the difficulty of expressing this channel in heterologous cell systems.

\section{Calcium channel mutant mice}

To address the pathogenesis of HypoPP caused by CACNA1S mutations, Wu and colleagues in the laboratory of Stephen Cannon report in this issue of the JCI the inves- 
A

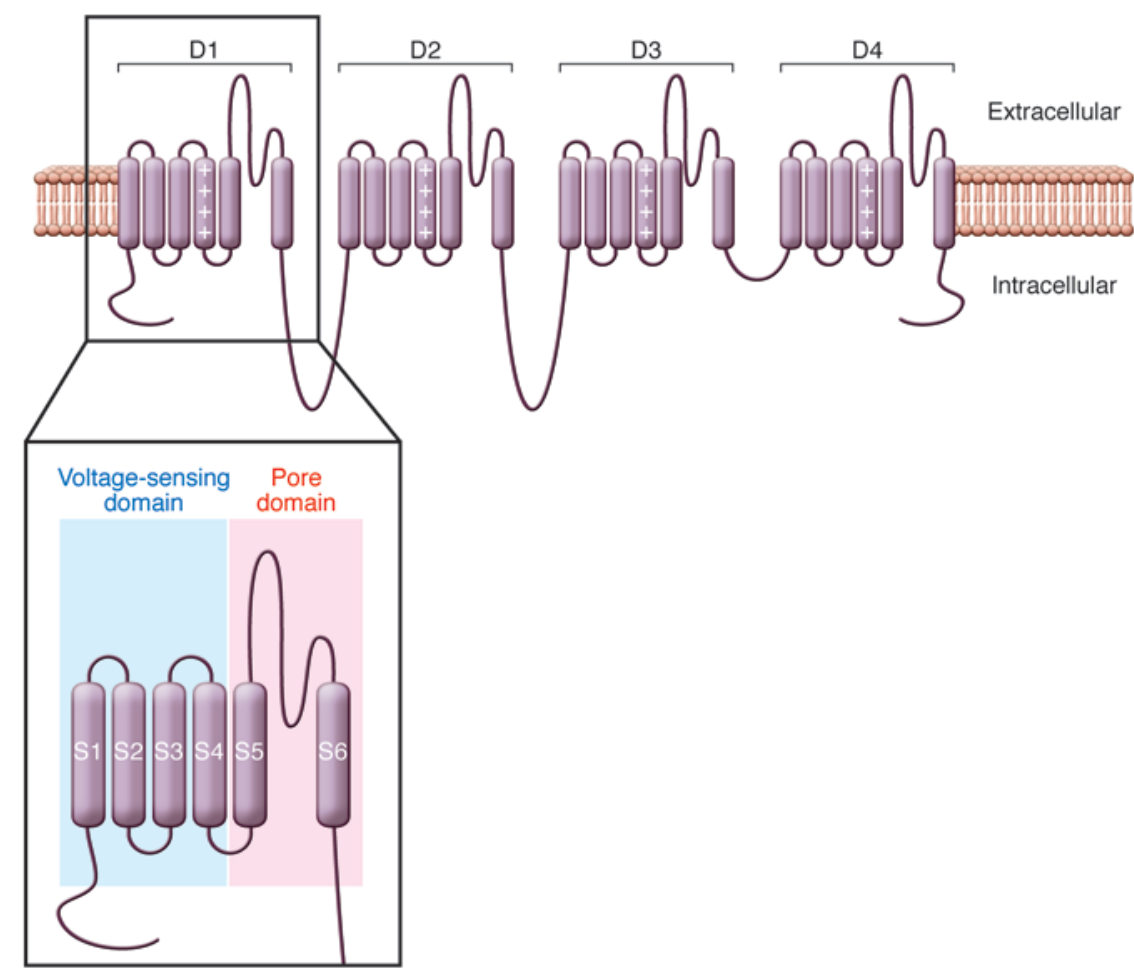

B

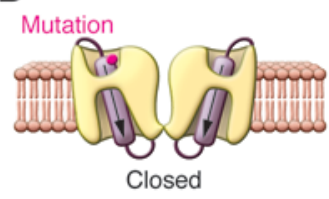

Depolarization

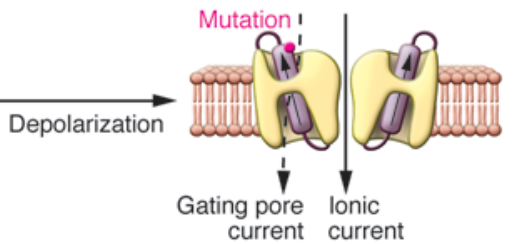

Figure 1

Voltage-gated sodium and calcium channel structural domains and location of gating pore. (A) Predicted transmembrane topology model of typical voltage-gated sodium or calcium channel. The S4 segments within voltage-sensing domains are indicated by a column of plus $(+)$ signs. The inset illustrates locations of the separate voltage-sensing and pore domains that are repeated four times in the channel protein. (B) A cutaway view showing the pathway through which ionic current or gating pore currents are conducted. The cylinders within the channels represent the S4 segments, and the approximate location of the HypoPP mutation Cav1.1 R528H is indicated. Figure modified from the Journal of General Physiology (23).

tigation of a novel knock-in mouse model of the disease (20). Mice were generated that express the most common human HypoPP mutation (Cav1.1 R528H), a histidine substitution for the outermost arginine residue of the D2/S4 segment in $\mathrm{Ca}_{V} 1.1$. Although animals did not exhibit spontaneous attacks of weakness, muscle strength was reduced more severely in male mice, consistent with the reduced penetrance in females observed for human HypoPP (6). Muscles from knock-in mice exhibited features previously observed in human HypoPP fibers, including reduced contractile force and paradoxical membrane depolarization evoked by low extracellular potassium or by glucose and insulin challenge. Muscle fibers from homozygous knock-in R528H mice exhibited a $-15-\mathrm{mV}$ depolarization of the resting membrane potential similar to human HypoPP fibers. In addition, these mice exhibited a chronic vacuolar myopathy similar to that observed in patients with this disorder.

A critical observation made by these investigators was the presence of an anomalous inward current in mutant mouse muscle fibers consistent with gating pore current rather than ionic current conducted through a known ion channel (20). These findings offered a mechanistic link between calcium channel mutation and attacks of muscle weakness. Anomalous gating pore current creates a precarious balance between the inward and outward currents that maintain the membrane potential. Factors such as hypokalemia that transiently depress potassium currents, which are required to maintain a normal resting membrane potential, can render the muscle severely depolarized and, consequently, inexcitable owing to inactivation of sodium channels.

Now that a common mechanism of evoking gating pore current has been established in HypoPP, several new and intriguing questions emerge. Can aberrant gating pore current be selectively blocked, and would this prevent attacks of paralysis? Do carbonic anhydrase inhibitors such as acetazolamide and dichlorphenamide, which are effective treatments for HypoPP regardless of genotype, affect gating pore current directly or indirectly? Are there triggering factors that act specifically by potentiating gating pore current? Finally, what is the basis for vacuolar myopathy, and why is there a sex-biased penetrance of mutations? These and other important questions can now be addressed using the mouse model developed by Wu and colleagues.

Although HypoPP was the first channelopathy for which aberrant gating pore current was implicated in disease pathogenesis, many other S4 mutations in voltage-gated sodium, calcium, and potassium channels have been found in other inherited disorders of membrane excitability, including epilepsy and cardiac arrhythmia syndromes (21). Are some of these conditions also "gating poreopathies"? A recent study demonstrating that an S4 segment mutation in the cardiac sodium channel $\left(\mathrm{Na}_{v} 1.5 \mathrm{R} 219 \mathrm{H}\right)$ associated with dilated cardiomyopathy evokes an aberrant proton current (22) suggests that this may be a more widespread phenomenon.

\section{Acknowledgments}

The author is supported by grants from the NIH (HL083374, NS032387).

Address correspondence to: Alfred L. George Jr., Division of Genetic Medicine, 529 Light Hall, Vanderbilt University, 2215 Garland Avenue, Nashville, Tennessee 37232-0275, USA. Phone: 615.936.2660; Fax:615.936.2661; E-mail: al.george@ vanderbilt.edu. 
1. George AL Jr. Inherited disorders of voltage-gated sodium channels. JClin Invest. 2005;115(8):1990-1999.

2. Jurkat-Rott K, Lehmann-Horn F. Muscle channelopathies and critical points in functional and genetic studies. J Clin Invest. 2005;115(8):2000-2009.

3. Meisler MH, Kearney JA. Sodium channel mutations in epilepsy and other neurological disorders. J Clin Invest. 2005;115(8):2010-2017.

4. Moss AJ, Kass RS. Long QT syndrome: from channels to cardiac arrhythmias. J Clin Invest. 2005 115(8):2018-2024

5. Jentsch TJ, Maritzen T, Zdebik AA. Chloride channel diseases resulting from impaired transepithelial transport or vesicular function. J Clin Invest. 2005;115(8):2039-2046.

6. Cannon SC, George AL. Pathophysiology of myotonia and periodic paralysis. In: Asbury AK, Mckhann GM, McDonald WI, Goadsby PJ, McArthur JC, eds. Diseases of the Nervous System, Clinical Neuroscience and Therapentic Principles. 3rd ed. Cambridge, United Kingdom: Cambridge University Press; 2002:1183-1206.

7. Rüdel R, Lehmann-Horn F, Ricker K, Kuther G Hypokalemic periodic paralysis: in vitro investigation of muscle fiber membrane parameters. Muscle Nerve. 1984;7(2):110-120.

8. Lehmann-Horn F, et al. Two cases of adynamia epi- sodica hereditaria: In vitro investigation of muscle cell membrane and contraction parameters. Muscle Nerve. 1983;6(2):113-121

9. Armstrong CM, Hille B. Voltage-gated ion channels and electrical excitability. Neuron. 1998; 20(3):371-380.

10. Swartz KJ. Sensing voltage across lipid membranes. Nature. 2008;456(7224):891-897.

11. Bezanilla F. How membrane proteins sense voltage. Nature Rev Mol Cell Biol. 2008;9(4):323-332.

12. Yang NB, George AL Jr, Horn R. Molecular basis of charge movement in voltage-gated sodium channels. Neuron. 1996;16(1):113-122.

13. Larsson HP, Baker OS, Dhillon DS, Isacoff EY. Transmembrane movement of the shaker $\mathrm{K}^{+}$channel S4. Neuron. 1996;16(2):387-397.

14. Starace DM, Stefani E, Bezanilla F. Voltage-dependent proton transport by the voltage sensor of the shaker $\mathrm{K}^{+}$channel. Neuron. 1997;19(6):1319-1327.

15. Sokolov S, Scheuer T, Catterall WA. Gating pore current in an inherited ion channelopathy. Nature. 2007;446(7131):76-78.

16. Sokolov S, Scheuer T, Catterall WA. Depolarization-activated gating pore current conducted by mutant sodium channels in potassium-sensitive normokalemic periodic paralysis. Proc Natl Acad Sci
US A. 2008;105(50):19980-19985.

17. Struyk AF, Cannon SC. A Na ${ }^{+}$channel mutation linked to hypokalemic periodic paralysis exposes a proton-selective gating pore. J Gen Physiol. 2007; 130(1):11-20.

18. Struyk AF, Markin VS, Francis D, Cannon SC. Gating pore currents in DIIS4 mutations of $\mathrm{Na}_{\mathrm{V}} 1.4$ associated with periodic paralysis: saturation of ion flux and implications for disease pathogenesis. J Gen Physiol. 2008;132(4):447-464.

19. Wu F, et al. A sodium channel knockin mutant (Nav1.4-R669H) mouse model of hypokalemic periodic paralysis. J Clin Invest. 2011;121(10):4082-4094.

20. Wu F, et al. A calcium channel mutant mouse model of hypokalemic periodic paralysis. $J$ Clin Invest. 2012;122(12):4580-4591.

21. Jurkat-Rott K, Groome J, Lehmann-Horn F. Pathophysiological role of omega pore current in channelopathies. Front Pharmacol. 2012;3:112.

22. Gosselin-Badaroudine P, et al. A Proton Leak current through the cardiac sodium channel is linked to mixed arrhythmia and the dilated cardiomyopathy phenotype. PLoS One. 2012;7(5):e38331.

23. Ahern CA, Horn R. Specificity of charge-carrying residues in the voltage sensor of potassium channels. J Gen Physiol. 2004;123(3):205-216.

\title{
aKlotho: FGF23 coreceptor and FGF23-regulating hormone
}

\author{
Harald Jüppner ${ }^{1}$ and Myles Wolf ${ }^{2}$
}

${ }^{1}$ Endocrine Unit and Pediatric Nephrology Unit, Massachusetts General Hospital, Boston, Massachusetts, USA. ²Division of Nephrology and Hypertension, Department of Medicine, University of Miami Miller School of Medicine, Miami, Florida, USA.

\begin{abstract}
Low levels of phosphate can disrupt bone ossification and predispose to fractures. FGF23 is one of the major determinants of phosphate homeostasis, acting to increase urinary phosphate excretion. However, the regulation of FGF23 is incompletely understood. In this issue of the JCI, Smith et al. show that the cleaved form of $\alpha$ Klotho, the membrane-bound form of which is an FGF23 coreceptor, serves as a novel endocrine regulator of phosphate homeostasis, capable of inducing FGF23 production in osteocytes.
\end{abstract}

Phosphate is a critical component of bone, and it serves numerous biological functions in the synthesis of DNA and membrane lipids, protein modifications, energy metabolism, and second messenger formation. FGF23 and parathyroid hormone (PTH) are the major regulators of phosphate homeostasis $(1,2)$. Both hormones increase urinary phosphate excretion by reducing proximal tubular expression of the sodi-

Conflict of interest: Myles Wolf has received research support or honoraria from Abbott Laboratories, Amgen, DiaSorin, Kai Pharmaceuticals, Luitpold Pharmaceuticals Inc., Mitsubishi, Sanofi, and Shire. Harald Jüppner has received honoraria from Kai Pharmaceuticals, Roche, Kyowa Hakko Kirin, Genzyme, Amgen, and Pfizer; he is a coinventor of a patent describing a technique to measure FGF23.

Citation for this article: J Clin Invest. 2012; 122(12):4336-4339. doi:10.1172/JCI67055. um-dependent phosphate cotransporters, NPT2a and NPT2c, thereby lowering serum phosphate levels. However, in contrast to FGF23, which decreases serum levels of 1,25-dihydroxyvitamin D (1,25D) by inhibiting renal $1 \alpha$-hydroxylase and stimulating 24-hydroxylase, PTH increases renal production of $1,25 \mathrm{D}$, which consequently enhances the absorption of phosphate (and calcium) from the intestinal tract (3).

FGF23 belongs to the subfamily of endocrine FGFs and mediates its phosphateregulating actions in the kidney through FGF receptors (FGFRs), most prominently FGFR1. These actions require the transmembrane form of $\alpha$ Klotho ( $\mathrm{mKL}$ ), which acts as the coreceptor that enhances the binding affinity of FGF23 to different FGFRs $(4,5)$. Ablation of $\alpha$ Klotho in mice leads to severe hyperphosphatemia, elevated levels of $1,25 \mathrm{D}$, hypercalcemia, diffuse soft tissue calcifications, accelerated aging, and premature death (6). These findings are largely indistinguishable from those observed in FGF23 loss-of-function models $(7,8)$.

Osteocytes are the primary cellular source of FGF23 after the fetal period (9), but little is known about their regulation and secretion. Although discovery of the molecular causes of monogenic hypophosphatemic rickets disorders has helped to identify a set of bone-derived proteins that decrease FGF23 production, the mechanisms of how these inhibitors suppress FGF23 remain largely unknown. Furthermore, despite our knowledge that changes in dietary phosphate intake can alter FGF23 levels in healthy individuals $(10,11)$, how serum phosphate or phosphate balance is sensed and how this signal is conveyed to the osteocyte to alter FGF23 expression remains completely unknown.

A novel regulator of FGF23 synthesis In this issue of the JCI, Smith et al. provide intriguing new findings about the 